\title{
AN INVARIANT MAY DRIVE THE DECISION TO ENCROACH AT UNSIGNALIZED INTERSECTIONS
}

\author{
Kip Smith ${ }^{1}$, Aurélie Thome ${ }^{2}$, Christian Blåberg ${ }^{1}$, Jonas Bärgman ${ }^{3}$ \\ ${ }^{1}$ Linköping University \\ Linköping Sweden \\ ${ }^{2}$ Insa de Lyon, France \\ ${ }^{3}$ Autoliv Research \\ Vårgårda, Sweden \\ Email:kipsm@ida.liu.se
}

\begin{abstract}
Summary: This paper introduces a novel approach to understanding when and where drivers make the Go / No Go decision (not) to turn left and encroach upon an approaching car that has the right-of-way in an unsignalized intersection. The source of data is approximately 2,400 hours of video recordings at two intersections near Göteborg, Sweden. Automated image processing software extracted the trajectories of the pairs of cars involved in more than 14,000 left turns across traffic at the first intersection and 2,400 at the second. We subdivided the data into four different left-turn scenarios - where the approaching car arrives from the opposite direction, from the lateral direction, from the intended direction (merging), and while making its own left turn. For each scenario, we found the distances between the turning car and the approaching car at the time when we can assume the decision (not) to turn is made and conducted logistic regressions to identify the distances associated with the 50/50 acceptance probabilities for the decision (not) to turn. We also calculated the resulting encroachment distances ('trailing buffers') for every decision to turn. We expected to find wide variability in these buffers. Instead, we observed separations that were virtually the same across scenarios at each intersection but differed across intersections. Tacit, intersection-dependent knowledge of this invariant may drive the decision of whether or not to turn and encroach. We discuss the implications this finding has for the design of in-vehicle active safety systems.
\end{abstract}

\section{INTRODUCTION}

Intersection crashes involving encroachment - a vehicle turning left across another's path - are over-represented in crash statistics and are frequently fatal (e.g., Chan, 2006). In response, the Swedish Road Administration and an industry consortium have participated in a multidisciplinary project directed at informing the design and development of in-vehicle active safety systems that would alert drivers of passenger cars to potential encroachments. This paper presents one set of analyses supported by that project.

The analyses focus on the influence of the separation between a pair of cars on the decision by the driver of one to turn left across the path of another that has the right-of-way. We call the car that turns left the 'provoker' because it may provoke an encroachment incident. We use the acronym 'POV' (principal other vehicle) for the car that is encroached upon. An 'incident' is a near-crash situation in which the gap between the provoker and the POV is small in both time 
and distance. The immediate goal of the study was to develop and demonstrate a novel methodology for quantifying how drivers define 'small in time and distance'.

Research on left-turns in intersections is active (e.g., Caird \& Hancock, 2002; Chan, 2006, 2007; Davis \& Swenson, 2004; Ragland et al., 2006; Yan \& Radwan, 2007; Yan, Radwan, \& Guo, 2007). Some studies use simulators to study driver actions in near-crash situations (e.g., Caird, Chisholm, Edwards \& Creaser, 2007). Most describe on-site observations of traffic and post-hoc analyses using logistic regression to identify the critical distance or critical time when $50 \%$ of drivers decide (not) to make the left turn. This study adopts and extends the latter approach. Most of the previous work has used time as the metric of separation (e.g., Tian, et al., 1999). Like Harrell and Spaulding (2001), we prefer to use distance as our principal metric. Information about distance is immediately available to both the driver's eye and the sensors that would inform in-vehicle systems. In contrast, time (e.g., time to contact) requires untenable assumptions about the constancy of driver intent and vehicle velocities.

This paper describes a new method for identifying the point where the provoker makes the decision (not) to turn left and encroach on a POV that has the right-of-way. We use that point to identify the distance between the provoker and the POV at the time when the decision is likely to be made and an alert might be warranted. No crashes were observed during the period of study.

\section{METHOD}

\section{Definition of lag and gap}

Many studies of left turns focus on gap acceptance - the situation where a provoker turns through a stream of traffic. The 'gap' is the measure of time or distance between vehicles in the stream. Ragland, et al., (2005) argue that in situations with only one POV and no stream of traffic, the appropriate term is 'lag' - the measure of time or distance between the opportunity to turn and the arrival of the POV. In this study, we have studied the lag, not the gap.

\section{The intersections}

Two non-signalized intersections were studied in this project. The first - at Sävenäs, $5 \mathrm{~km}$ east of Göteborg, Sweden - is a 3-way intersection in a semi-rural industrial area with significant traffic flow and a $50 \mathrm{kph}$ speed limit. The second - at Jung, $120 \mathrm{~km}$ northeast of Göteborg - is a 4-way $70 \mathrm{kph}$ intersection in a fully rural area on the main road from Göteborg to Stockholm (E20). Traffic on the E20 often exceeds $100 \mathrm{kph}$. There were four major constraints on the selection process that settled upon the Sävenäs and Jung intersections: camera stability, ease of access, estimates of relatively high incident rates, and proximity to either Vårgårda or Göteborg.

\section{Automated image processing of video data}

Digital video recording equipment was installed in both intersections. Approximately 1800 (600) hours of video recordings were collected by roof-top cameras at Sävenäs (Jung). An automated trajectory extraction algorithm (Hedborg \& Johansson, 2008) extracted time series of the positions, headings, velocities, and estimated sizes and orientations of vehicles within $80 \mathrm{~m}$ of the 
intersections at $20 \mathrm{~Hz}$. The image processing system classified the trajectories of more than $106,000(33,000)$ vehicles at Sävenäs (Jung), Table 1. These totals reflect a filtering that eliminated all over-size vehicles, e.g., trucks and buses, to retain our focus on passenger cars. The time-stamped trajectory data make it possible to identify pairs of cars that were in the intersection at the same time and to identify which car turned left (or not) across the path of another and whether there were additional cars in view.

Table 1. Number and relative frequency of encroachments

\begin{tabular}{|c|c|c|c|c|c|c|c|c|}
\hline \multirow[b]{3}{*}{ Scenario } & \multicolumn{4}{|c|}{ Sävenäs } & \multicolumn{4}{|c|}{ Jung } \\
\hline & \multicolumn{2}{|c|}{$\mathrm{No} \mathrm{Go}+\mathrm{Go}$} & \multicolumn{2}{|c|}{ Go } & \multicolumn{2}{|c|}{$\mathrm{No}$ Go + Go } & \multicolumn{2}{|c|}{ Go } \\
\hline & $\mathrm{N}$ & $\%$ & $\mathrm{~N}$ & $\%$ & $\mathrm{~N}$ & $\%$ & $\mathrm{~N}$ & $\%$ \\
\hline LTAP/OD & 4650 & 4.3 & 865 & 18.6 & 1041 & 3.1 & 171 & 16.4 \\
\hline LTAP/LD & 2022 & 1.9 & 365 & 18.1 & 785 & 2.3 & 65 & 8.7 \\
\hline Crossing & 5279 & 4.9 & 709 & 13.4 & 71 & 0.2 & 10 & 14.1 \\
\hline Merging & 2590 & 2.4 & 243 & 9.4 & 535 & 1.6 & 54 & 10.1 \\
\hline All traffic & 106913 & 13.6 & 2182 & 2.0 & 33824 & 7.2 & 303 & 0.9 \\
\hline
\end{tabular}

\section{The four traffic scenarios}

The analyses considered four different traffic scenarios separately. They are sketched in Figure 1. In each scenario the white car has the right-of-way and is the POV. The provoker is the black car. Its driver makes the decision of whether or not to turn left in front of the POV. Case A is a left turn across path from the opposite direction (LTAP/OD). The provoker turns from the main road but should yield to the POV. In the other three cases, the provoker turns from the secondary road where there are yield (stop) signs at Sävenäs (Jung). Case B represents a left turn left across the right-of-way path from the lateral direction (LTAP/LD). In crossing case $C$, both cars are turning left with the POV turning from the right-of-way road. In merging Case D, both cars intend to travel in the same direction down the right-of-way road. The lists of 'No Go + Go' in Table 1 represent observed opportunities to encroach. The lists of 'Go' represent decisions to turn left. Inspection of Table 1 reveals that the base rate of encroachment is low, approximately $2 \%(1 \%)$ of all observed traffic scenarios at Sävenäs (Jung). The higher speeds at and greater width of the Jung intersection may explain the two-fold disparity in observed frequencies.

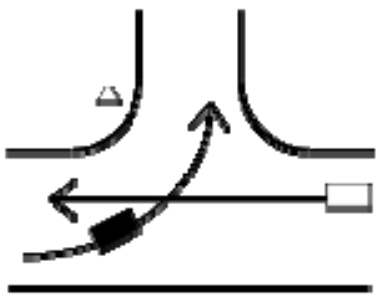

(a)

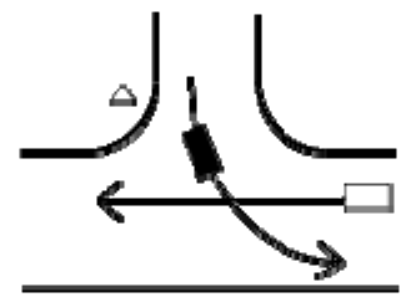

(b)

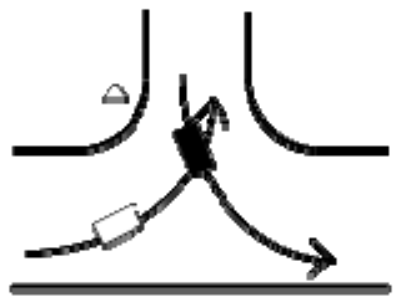

(c)

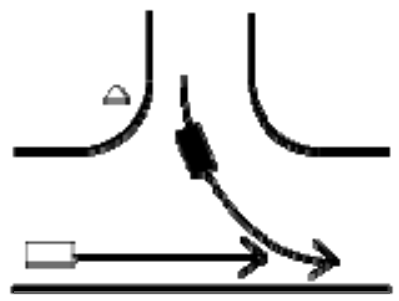

(d)

Figure 1. The four traffic scenarios: (a) LTAP/OD, (b) LTAP/LD, (c) crossing, (d) merging 


\section{Minimum velocity marks the decision point}

The novel method we introduce here is based on the premise that the driver of the turning car the provoker - makes the decision to encroach at the time when his or her velocity is at its minimum. This line of reasoning maintains that the driver begins to accelerate as soon as the decision to go is made. If the decision is not to go, deceleration continues. If the car stops, its location defines its minimum velocity point. We examined the image data to identify the locations of velocity minima of all observations listed in Table 1 and to compute their distribution for each scenario. At Sävenäs, the median minima was $8.9 \mathrm{~m}$ from the center of the intersection on both roads. At Jung, the median minima ranged from 8.0 to $12.5 \mathrm{~m}$. The significance of these points is straightforward - they represent our best estimate of where the driver of a provoker is likely to make the decision (not) to encroach on the POV. This point defines the center of a short but contextually significant zone where an alert to an impending encroachment incident is likely to have the greatest effect on a provoker's decision making.

\section{Lag distance and time at the decision point}

The separation between a provoker and the POV at the decision point defines the lag at the point where and time when we infer that most drivers make the decision (not) to encroach. The data generated by the image processing system make it possible to compute the lag as functions of both distance and time. We calculated both metrics of lag for all observations of decisions (not) to encroach. We used logistic regression to calculate the '50/50 point' in the distribution of lag distances and times. This value represents the distance at which $50 \%$ of drivers decide to encroach and 50\% do not. Tables 2 and 3 summarize the 50/50 points as functions of both time and distance for Sävenäs and Jung, respectively. When lags are shorter than these thresholds, most potential provokers are not willing to encroach.

Table 2. Summary of logistic regression models for encroachment decisions at Sävenäs

\begin{tabular}{lcccc}
\hline & & \multicolumn{3}{c}{ Scenario } \\
Separation at 50/50 point & LTAP/OD & LTAP/LD & Crossing & Merging \\
\cline { 2 - 5 } Meters & 54.5 & 69.2 & 63.1 & 67.7 \\
Seconds & 4.75 & 5.93 & 7.75 & 6.05 \\
Relative velocity (kph) & 41 & 42 & 29 & 40 \\
Trailing buffer (meters) & 57.7 & 55.5 & 49.9 & 46.0 \\
\hline
\end{tabular}

Table 3. Summary of logistic regression models for encroachment decisions at Jung

\begin{tabular}{lccc}
\hline & & Scenario & \\
Separation at 50/50 point & LTAP/OD & LTAP/LD & Merging \\
\cline { 2 - 4 } Meters & 125 & 130 & 110 \\
Seconds & 5.6 & 6.3 & 5.0 \\
Relative velocity (kph) & 80 & 74 & 79 \\
& & & 100 \\
Trailing buffer (meters) & 144 & 132 & 100 \\
\hline
\end{tabular}




\section{Post-encroachment distance}

Post-encroachment distance is the minimum distance between the provoker and POV during the encroachment. It can be calculated only when the provoker's decision was to Go and there was, in fact, an encroachment. It is a direct measure of the outcome of the provoker's decision to encroach. Our computation of post-encroachment considers the location of the cars where their trajectories cross and their dimensions to provide an estimate of just how 'near' the near-crash situation became. The analysis considered only pairs of vehicles that were within $15 \mathrm{~m}(37.5 \mathrm{~m})$ of the center of the intersection at Sävenäs (Jung).

\section{RESULTS}

\section{Distance, time, and apparent velocity at the decision point}

As shown in Tables 2 and 3, the 50/50 point for the lag between the provoker and the POV varies across the four scenarios. At Sävenäs, the direction of approach appears to influence the driver's decision. The distance is much less $(54.5 \mathrm{~m})$ for the oncoming scenario (LTAP/OD) than it is for the three cases where the provoker enters from the secondary road $(66 \pm 3 \mathrm{~m})$. This directional dependence is not replicated at Jung. It is not possible to ascertain whether the apparent lack of directional dependence at Jung is due to the relatively small sample size or to contextual factors associated with the greater width of the intersection and the higher speeds of traffic on the E20. The width and speed undoubtedly influence the doubling of the observed values. Tables 2 and 3 also list the 50/50 points for the time in seconds between vehicles at the decision point. The ratio of the 50/50 points by distance and time is an estimate of the apparent relative velocity of the two cars at the time when the driver of the provoker decides (not) to encroach. With the exception of the one crossing scenario, the velocities are remarkably uniform at both Sävenäs $(40 \mathrm{kph})$ and Jung (80 kph).

\section{Post-encroachment distance}

We calculated the post-encroachment distance for each observed Go decision. The data from all scenarios are distributed normally. The means of the distributions represent our best estimate of the 'trailing buffers' - the outcome of the decision to encroach. They are listed in the bottom rows of Tables 2 and 3. We expected to find wide variability in these distances. Instead, we observed a relatively narrow range of outcomes across all four scenarios at Sävenäs ( $52 \pm 6$ meters) and the two non-merging scenarios at Jung (138 \pm 6 meters). At both Sävenäs and Jung, the longest buffer is for the LTAP/OD scenario and the shortest for the merging scenario.

\section{DISCUSSION}

These data support the inference that drivers make contextually sensitive decisions of whether or not to encroach. At both Sävenäs and Jung, the 50/50 points in both time and distance are shorter for the LTAP/OD scenario than in the LTAP/LD scenario. This difference may be explained by the observation that the provoker in the OD scenario often has some velocity heading into the intersection. Inertia may reinforce a Go decision. 
With the exception of the Sävenäs crossing scenario, the values of the apparent relative velocity at the decision point are remarkably constant - $40 \mathrm{kph}$ at Sävenäs and $80 \mathrm{kph}$ at Jung. The uniformity of the implied velocities suggests that the drivers in our study may share a tacit (unspoken but understood) expectation for the velocity of vehicles at these intersections. Tacit expectations for velocity are a hallmark of ecological psychology and its interpretation of driver behavior (e.g., Caird and Hancock, 2002). Our data strongly support the argument that drivers act as if they are able to extract information about the velocity of traffic directly from the optic flow field. This finding supports the contention that it would be appropriate for designers of active systems to assume that drivers share tacit and contextual-sensitive expectations for the velocity of traffic.

The values of the trailing buffers are remarkably uniform as well, on the order of 60 meters at Sävenäs and 140 meters at Jung. This internal consistency, if it is found to hold elsewhere, may be a key finding. It is possible that the drivers of the 17,000+ pairs of vehicles in this study acted as if they knew how much separation they needed to have at the decision point to achieve a target and 'safe' trailing buffer. This implies tacit knowledge of an invariant relationship between three parameters: the decision point (the point of minimum velocity), the distance to POV at that point, and the expected velocity profiles of both vehicles. All three parameters in this hypothesized invariant relationship are amenable to implementation in in-vehicle active safety systems.

This paper has suggested a novel approach to addressing the hazard to public safety posed by encroachment at unsignalized intersections. We suggest that the median of the distribution of velocity minima for the turning cars represents our best estimate of where potential provokers make the decision (not) to encroach. It may also be the point where a warning (of some unspecified kind) about the potential for encroachment may have its greatest influence.

Our analyses found that the ratios of the 50/50 points in distance and time define relatively constant velocities - $40 \mathrm{kph}$ at Sävenäs and $80 \mathrm{kph}$ at Jung. This finding was somewhat expected. In contrast, we did not expect to observe consistency in the length of the trailing buffers generated by provokers who decide to encroach. It appears that the drivers in our study share tacit expectations for both the relative velocities of cars in intersection and the outcomes of their decisions to encroach. The buffer length, $60 \mathrm{~m}$ at Sävenäs and $\sim 140 \mathrm{~m}$ at Jung, represents an outcome that provokers appear to find acceptable. In sum, these data and this method of analysis have the potential to inform the design of active safety systems that address encroachments at unsignalized intersections.

\section{ACKNOWLEDGEMENTS}

This work was supported by Autoliv AB, SAAB AB, Volvo Car Corporation, and the Swedish Road Administration. All opinions are the authors' and may not be endorsed by the sponsors.

\section{REFERENCES}

Caird, J. K., Hancock, P. A. (2002). Left turn and gap acceptance accidents. In: Dewar, R.E., Olson, R. (Eds.), Human Factors in Traffic Safety. Lawyers \& Judges Publishing, Tucson, AZ, pp. 591-630. 
Caird, J. K., Chisholm, S. L. Edwards, C. J., \& Creaser, J. I. (2007). The effect of yellow light onset time on older and younger drivers' perception response time (PRT) and intersection behavior. Transportation Research Part F, 10, 383-396.

Chan, C.-Y. (2006). Characterization of driving behaviors based on field observation of intersection left-turn across-path scenarios. IEEE Transactions on Intelligent Transportation Systems, 7 (3), 322-331.

Chan, C.-Y. (2007). An investigation of traffic characteristics and their effects on driver behaviors in intersection crossing-path maneuvers. Proceedings of the 2007 IEEE Intelligent Vehicles Symposium.

Davis, G. A. \& Swenson, T. (2004). A field study of gap acceptance by left-turning drivers. Transportation Research Record, 1899, 71-75.

Harrell, W. A. \& Spaulding, L. M. (2001). Social psychological models of choice behavior and drivers' left turns. Journal of Social Psychology, 141, 714-722.

Hedborg, J., \& Johansson, B. (2008). Real time camera ego-motion compensation and image rectification on GPU. Real-time image processing.

Ragland, D. R., Arroyo, S., Shladover, S. E., Misener, J. A., \& Chan, C.-Y. (2006). Gap acceptance for vehicles turning left across on-coming traffic: Implications for intersection decision support design. Proceedings of the Transportation Research Board 2006 Annual Meeting, Paper No. 06-2696.

Tian, Z., Vandehey, M., Robinson, B. W., Kittelson, W., Kyte, M., Troutbeck, R., Brilon, W., \& Wu, N. (1999). Implementing the maximum likelihood methodology to measure a driver's critical gap. Transportation Research Part A, 33, 187-197.

Yan X., \& Radwan, E. (2007). Effect of restricted sight distances on driver behaviors during unprotected left-turn phase at signalized intersections. Transportation Research Part F, 10, 330-344.

Yan, X., Radwan, E. \& Guo, D. (2007). Effects of major-road vehicle speed and driver age and gender on left-turn gap acceptance. Accident Analysis \& Prevention, 39 (4), 843-852. 